\title{
Benign Post-Radiation
}

Rectal Stricture Treated with Endoscopic Balloon Dilation and Intralesional Triamcinolone Injection

Michael Karanikas ${ }^{a}$ Panagiotis Touzopoulos $^{a}$

Alexandros Mitrakas ${ }^{a}$ Petros Zezos ${ }^{b}$ Paul Zarogoulidis ${ }^{c}$ Nikolaos Machairiotis ${ }^{d}$ Eleni Efremidou $^{a}$

Nikolaos Liratzopoulos ${ }^{a}$ Alexandros Polychronidis ${ }^{a}$ George Kouklakis ${ }^{b}$

${ }^{\mathrm{a}}$ 1st Department of Surgery and ${ }^{\mathrm{b}}$ Gastrointestinal Endoscopy Unit, Democritus University of Thrace, University General Hospital of Alexandroupolis, Alexandroupolis, 'Pulmonary Department, 'G. Papanikolaou' General Hospital, Aristotle University of Thessaloniki, Thessaloniki, and ${ }^{\mathrm{d}}$ Surgery Department (NHS), University General Hospital of Alexandroupolis, Alexandroupolis, Greece

\section{Key Words}

Post-radiation stricture $\cdot$ Balloon dilation - Triamcinolone injection $\cdot$ Chronic radiation proctitis

\begin{abstract}
Post-radiation stricture is a rare complication after pelvis irradiation, but must be in the mind of the clinician evaluating a lower gastrointestinal obstruction. Endoscopy has gained an important role in chronic radiation proctitis with several therapeutic options for management of intestinal strictures. The treatment of rectal strictures has been limited to surgery with high morbidity and mortality. Therefore, a less invasive therapeutic approach for benign rectal strictures, endoscopic balloon dilation with or without intralesional steroid injection, has become a common treatment modality. We present a case of benign post-radiation rectal stricture treated successfully with balloon dilation and adjuvant intralesional triamcinolone injection. A 70-year-old woman presented to the emergency room complaining for 2 weeks of diarrhea and meteorism, 11 years after radiation of the pelvis due to adenocarcinoma of the uterus. Colonoscopy revealed a stricture at the rectum and multiple endoscopic biopsies were obtained from the stricture. The stricture was treated with endoscopic balloon dilation and intralesional triamcinolone injection. The procedure
\end{abstract}


appears to have a high success rate and a very low complication rate. Histologic examination of the biopsies revealed non-specific inflammatory changes of the rectal mucosa and no specific changes of the mucosa due to radiation. All biopsies were negative for malignancy. The patient is stricture-free 12 months post-treatment.

\section{Introduction}

Radiotherapy has become one of the most important treatment options for malignancy. Tumors of the pelvis are increasingly being irradiated for local treatment benefit, with the subsequent complication of anorectal injury. Tumors inside the pelvic cavity are suited to receiving radiotherapy as the exposure to healthy tissue can be minimized and high doses can be delivered to the target tumor [1]. However, the proximity of the rectum to the back of the prostate, the cervix and the uterus makes it anatomically impossible for the rectum not to receive radiation while irradiating these neighbor organs [2]. The term radiation enteritis refers to radiation injury of the colon and small intestine mucosa characterized by telangiectasias, pallor and friability, while the injury or the rectum due to that cause is referred to as radiation proctitis [2]. Radiation proctitis is usually a result of pelvic irradiation for malignancies of the prostate, cervix, uterus, anus, rectum or urinary bladder and can be subdivided into two types: acute and chronic. Acute colitis is typically seen within a few days to 6 weeks after radiation. Chronic colitis typically presents within 9-12 months with rectal bleeding or obstructed defecation due to strictures [2]. In addition, these patients seem to be at increased risk of developing rectal cancer [3]. Historically, treatment of rectal strictures has been limited to surgery with a high morbidity and mortality. Therefore, a less invasive therapeutic approach for benign rectal strictures, endoscopic balloon dilation with or without intralesional steroid injection, has become a common method of treatment [4]. We present a case of benign post-radiation rectal stricture treated successfully with balloon dilation and adjuvant intralesional triamcinolone injection.

\section{Case Report}

A 70-year-old woman presented to the emergency room complaining of 2 weeks of diarrhea and meteorism. She had been operated for adenocarcinoma of the uterus 11 years before and radiation therapy had been performed for 1 month post-hysterectomy. She had also been operated for breast cancer 9 years earlier. The regular post-operation follow-up with thorax, abdominal and pelvis computed tomography (CT) had been unremarkable. She also suffered from type 2 diabetes, hypertension and hyperlipidemia and was receiving oral medication (glimepiride $4 \mathrm{mg}$ once daily $1 \times 1$, sitagliptin $(50 \mathrm{mg})+$ metformin $(1,000 \mathrm{mg}) 1 \times 1$, perindopril arginine $5 \mathrm{mg} 1 \times 1$, nebivolol $5 \mathrm{mg} 1 \times 1$, and simvastatin $20 \mathrm{mg} 1 \times 1$ ). On physical examination the patient was mildly dehydrated with a blood pressure of $155 / 85 \mathrm{~mm} \mathrm{Hg}$ and a heart rate of $98 / \mathrm{min}$. Her abdomen was distended with tenderness, without sings and findings of acute abdomen. A stricture was palpated $10 \mathrm{~cm}$ from the anus into the rectum at digital rectal examination. Complete blood count revealed a normal leukocyte count (white blood cells 8,200/ $\mathrm{ll}$ ) with mild neutrophilia (80.1\%), hemoglobin $13 \mathrm{~g} / \mathrm{dl}$, hematocrit $39.6 \%$, and platelets $305,000 / \mu$ l. Serum biochemical profile revealed hyperglycemia $(277 \mathrm{mg} / \mathrm{dl})$, normal renal function (urea $40 \mathrm{mg} / \mathrm{dl}$, creatinine $0.6 \mathrm{mg} / \mathrm{dl}$ ) and a C-reactive protein level of $1.35 \mathrm{mg} / \mathrm{dl}$. Plain abdominal radiographs revealed moderate diffuse colonic distention, without evidence of complete obstruction. Abdominal CT was unremarkable except for colonic distention.

Colonoscopy revealed a stricture $10 \mathrm{~cm}$ from the anus, reducing the rectal lumen to $5 \mathrm{~mm}$ in

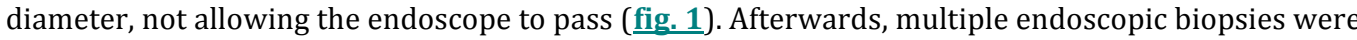


obtained from the stricture; a deflated $17 \mathrm{~mm}$ guidewire-bearing dilating balloon, $5.5 \mathrm{~cm}$ long (Rigiflex, Boston Scientific, Nattick, Mass., USA), was passed through the endoscope and was advanced through the stricture over the guidewire under direct endoscopic guidance. The balloon was inflated to maximum pressure according to the manufacturer's instructions (fig. 2). Mild bleeding with no evidence of perforation was noted following removal of the balloon. After the dilation, the endoscope could be advanced through the stricture, which was $6 \mathrm{~cm}$. The rectal and colonic mucosa proximal to the stricture appeared normal (fig. 3). After satisfactory dilation had been achieved, triamcinolone ( $2 \mathrm{ml}$ of $40 \mathrm{mg} / \mathrm{ml}$ diluted to $10 \mathrm{ml}$ with normal saline) was injected quadrantically into the stricture using a varix injection catheter with a $5 \mathrm{~mm}$ needle. Quadrantic injection was repeated at $2 \mathrm{~cm}$ intervals. The procedure was uneventful; the post-procedure abdominal X-ray was negative for pneumoperitoneum and the patient was discharged after $24 \mathrm{~h}$ without any treatment. One week later flexible endoscopy showed successful and sustained dilation of the stricture. Histologic examination of the biopsies revealed non-specific inflammatory changes of the rectal mucosa and no specific changes of the mucosa due to radiation. All biopsies were negative for malignancy. The patient is stricture-free 12 months post-treatment as confirmed by repeated flexible endoscopy.

\section{Discussion}

Rectal strictures are chronic narrowings of the intestinal lumen by abnormal tissue, resulting in complete or partial bowel obstruction [5]. This tissue may be scar tissue resulting from chronic rectal or anal inflammation, trauma, foreign bodies, prior surgery, perianal fistulas or pelvic irradiation [5].

'Radiation enteritis' refers to injury of the colon and small intestine after radiation and can be subdivided into acute and chronic. For radiation injuries of the rectum, the term 'radiation proctitis' is used. In acute radiation enteritis, histopathological changes of intestinal mucosa are inflammatory cell infiltrate, reduced crypt micro-abscesses, epithelial denudation and ulceration [6]. Patients with acute radiation enteritis or proctitis present diarrhea, abdominal pain or rectal bleeding shortly after a course of radiotherapy, but can be presented within 3 months [7]. Histologic features of chronic radiation enterocolitis are obliterative endarteritis, lymphatic dilation, tissue ischemia and necrosis, submucosal fibrosis and collagen deposition, which can lead to severe transmural fibrosis and formation of strictures or fistulas [6]. Chronic radiation colitis has been reported in up to $20 \%$ of patients receiving radiation in the pelvis, and the rate of intestinal obstruction due to strictures is referred to as 1-15\% [6]. There are also patient and treatment factors which may increase the risk of developing chronic radiation colitis. These factors include low body mass index, co-morbid conditions (diabetes, hypertension, collagen vascular diseases, inflammatory bowel disease), smoking, volume of bowel inside the radiation field, radiotherapy dose, radiotherapy technique and simultaneous chemotherapy [6].

The clinician should have chronic radiation colitis in mind while evaluating patients with bowel obstruction after radiation therapy, especially in the pelvis. Symptoms range from abdominal pain and vomiting to diarrhea and flatulence [7]. Patients affected by post-radiation strictures display obstructing symptoms, including diarrhea or tenesmus often producing narrow ribbons of feces or no feces at all. Abdominal pain and vomiting may occur in response to a stricture, but combined with a history of weight loss can also occur secondary to recurrent neoplasia. Laboratory tests investigating the evidence of anemia may be helpful. The importance of this was presented by Andreyev et al. [8], who reported the incidence of new or recurrent neoplasia with gastrointestinal symptoms 2 years after radiation of the pelvis in 
around $12 \%$ of patients, making this a common diagnosis. Patients with colonic symptoms must be investigated with colonoscopy, while patients with non-specific gastrointestinal symptoms should be investigated with CT with or without enteroclysis, looking for a tumor. In our patient, CT was unremarkable, while histopathology of biopsy specimens from the stricture during colonoscopy revealed inflammatory mucosal changes, compatible with but not specific for chronic radiation colitis, and was negative for malignancy.

Traditionally, surgical techniques, such as line rectal pull-through, myotomy or stricturoplasty, have been performed to manage rectal strictures [5]. However, post-operative complications can occur in up to $30 \%$, around half of the patients will require more than one surgery [9], and one third of them die in hospital [10]. Surgery in these patients is complicated because of the extensive fibrosis and adhesions in the pelvis and should be avoided if possible.

Endoscopy has recently gained an important role in chronic radiation colitis, with therapeutic options such as argon plasma coagulation for angiodysplastic lesions and balloon dilation for management of intestinal strictures [6]. Endoscopic placement of self-expanding metallic stents has been used for management of malignant strictures, alternatively to surgery. The success rate was extremely high ( $>92 \%)$ and reported a complication rate $<5 \%$ [11]. There is also a case report in which a radiation stricture of the rectosigmoid was successfully treated with self-expanding metallic stents, and obstructing symptoms were relieved in a patient who lived without any complications for 4 months until he died of sepsis due to pneumonia and bedsores [12].

Endoscopic balloon dilation is effective for managing both surgical anastomotic strictures and inflammatory disease strictures, with an up to $97 \%$ success rate and major complications $<3 \%$ [13]. In a systematic review, Hassan et al. [14] presented the results of 13 studies enrolling 347 patients treated with balloon dilation due to post-surgical anastomotic Crohn's strictures. It is reported that 58\% of the patients avoided surgery until 36 months of follow-up, while $59 \%$ of them needed a single session, $22 \%$ required 2 sessions, and 19\% required more than 2 sessions with range from 3 to 18. Results for post-radiation strictures appear to be the same. In a recent research, Hu et al. [15] presented the results of 17 patients who underwent balloon dilation due to pharyngoesophageal strictures caused by radiotherapy of the head and neck. Clinical success was achieved in $64.7 \%$ of patients with mean range of 2.4 sessions (range 1-7 sessions).

Moreover, intralesional steroid injection has been used together with balloon dilation to treat patients with colorectal anastomotic strictures or strictures secondary to Crohn's disease. Adjuvant intralesional steroid injection is safe and only adds a few minutes in procedure time. The exact mechanism by which intralesional steroids prevent reformation of the strictures is unknown. Hassan et al. [14], in a study of dermal keloids, reported that triamcinolone seems to inhibit collagen formation, enhance collagen breakdown and prevent cross-linking of collagen that results in scar contracture. Gastrointestinal strictures are histologically similar to scars. Intralesional use of triamcinolone appears to enhance the effect of balloon dilation and even prolong the relapse interval [14]. No major complications have been reported yet. 


\section{Conclusion}

Post-radiation stricture is a rare complication after pelvis irradiation, but must be in the mind of the clinician evaluating a lower gastrointestinal obstruction. Colonoscopy plays an important role in the diagnosis and exclusion of malignancy. Endoscopic balloon dilation with adjuvant intralesional steroid injection can be a minimally invasive, safe and effective treatment option for the management of post-radiation benign rectal strictures. The procedure appears to have a high success rate and a very low complication rate. An alternative treatment option is the endoscopic placement of a metallic stent in the stricture area, and last choice surgery, a procedure with very high morbidity and mortality rates.

\section{Disclosure Statement}

The authors have no conflicts of interest to declare.

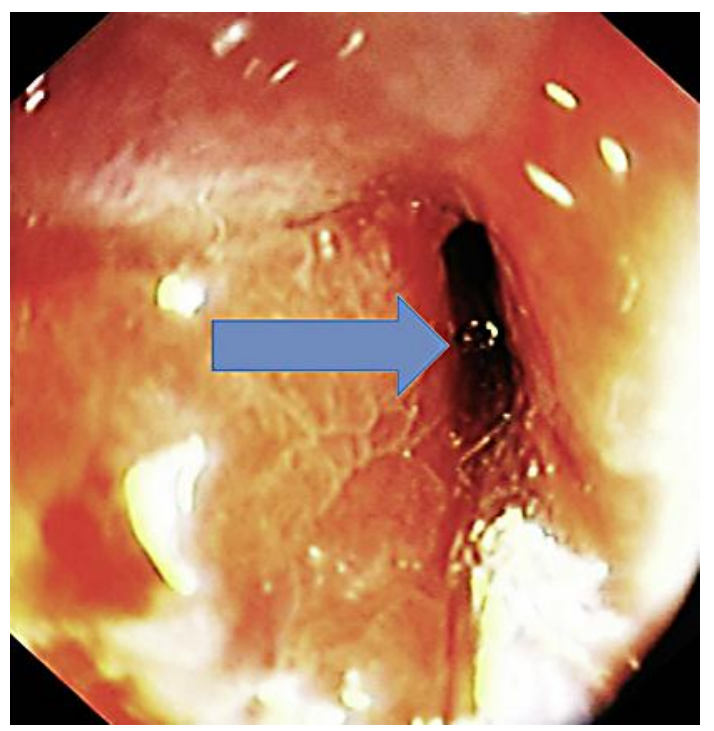

Fig. 1. Rectal stricture $10 \mathrm{~cm}$ from the anus, not allowing the endoscope to pass (blue arrow). 

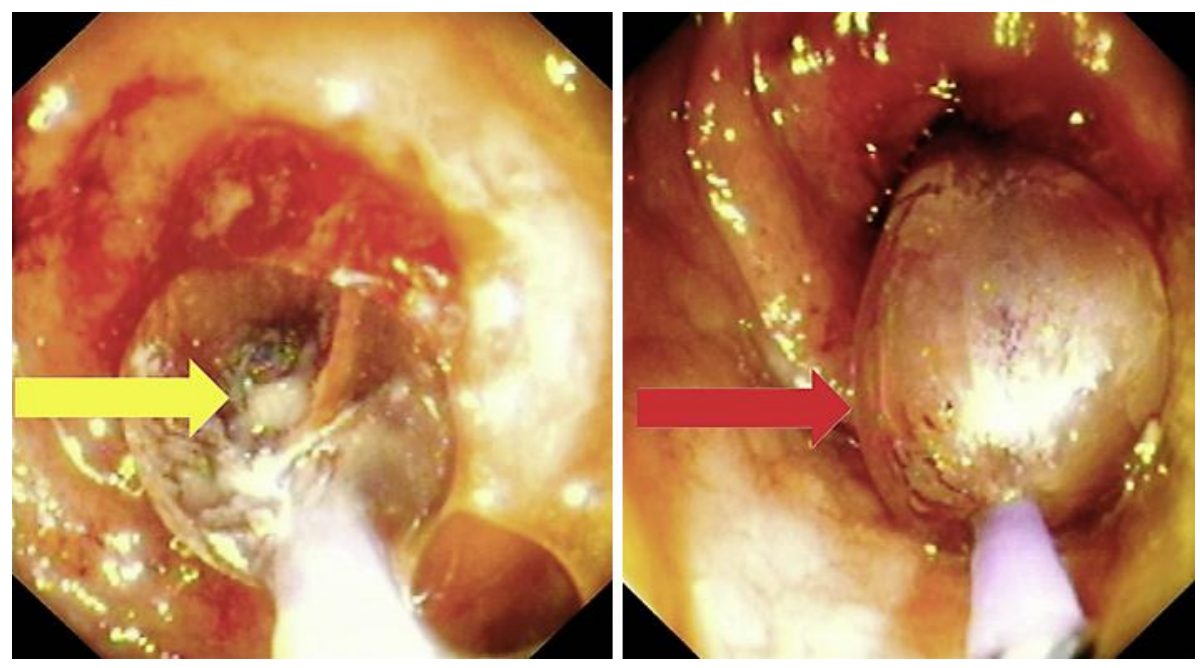

Fig. 2. A deflated $17 \mathrm{~mm}$ guidewire-bearing dilating balloon, $5.5 \mathrm{~cm}$ long (Rigiflex, Boston Scientific, Nattick, Mass., USA) was passed through the endoscope and advanced through the stricture over the guidewire under direct endoscopic guidance (yellow arrow). The balloon was inflated to maximum pressure according to the manufacturer's instructions (red arrow).
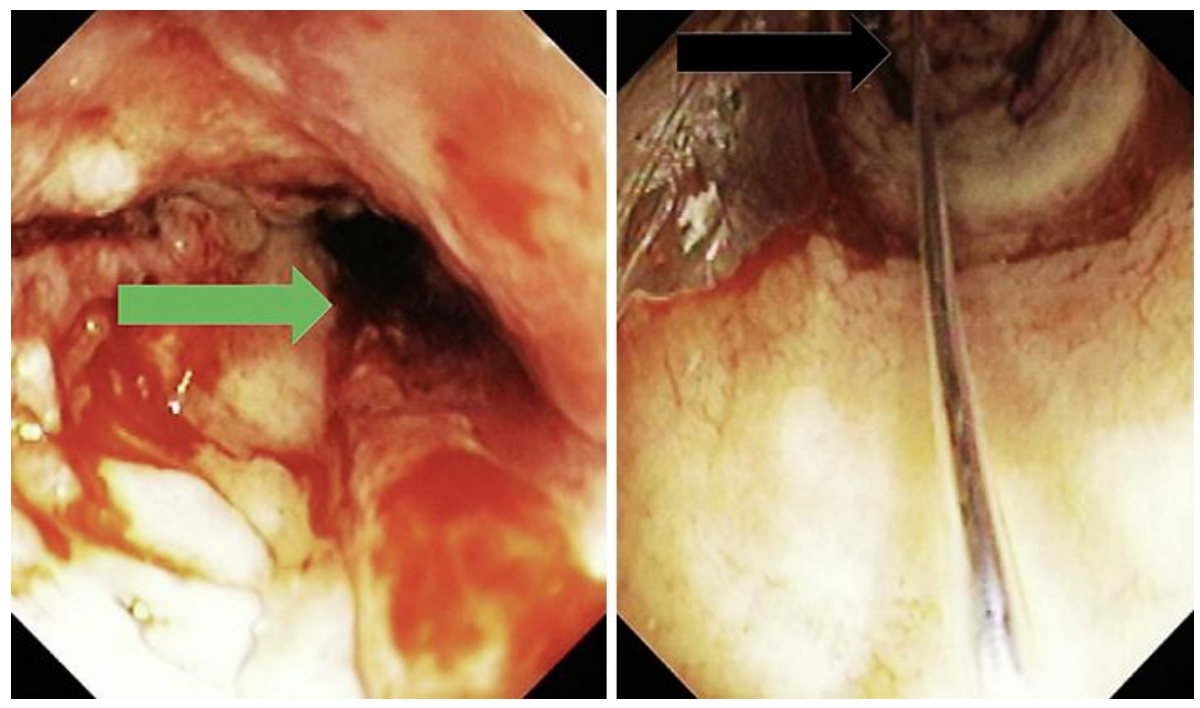

Fig. 3. Stricture after balloon dilation (green arrow) and area proximal to the stricture (black arrow). 


\section{References}

1 Kyriazi MA, Stafyla VK, Kondi-Pafiti A, Arkadopoulos N, Dafnios N, Hasiakos D, Fotiou S, Mastorakos D, Smyrniotis V: A novel technique for surgical reconstruction of the perineal floor following anteroposterior exenteration of the pelvis - case report and review of the literature. Eur J Gynaecol Oncol 2010;31:201-205.

2 Do NL, Nagle D, Poylin VY: Radiation proctitis: current strategies in management. Gastroenterol Res Pract 2011;2011:917941.

-3 Kimura T, Iwagaki H, Hizuta A, Nonaka Y, Tanaka N, Orita K: Colorectal cancer after irradiation for cervical cancer - case reports. Anticancer Res 1995;15:557-558.

-4 Singh VV, Draganov P, Valentine J: Efficacy and safety of endoscopic balloon dilation of symptomatic upper and lower gastrointestinal Crohn's disease strictures. J Clin Gastroenterol 2005;39:284-290.

-5 Schlegel RD, Dehni N, Parc R, Caplin S, Tiret E: Results of reoperations in colorectal anastomotic strictures. Dis Colon Rectum 2001;44:1464-1468.

-6 Theis VS, Sripadam R, Ramani V, Lal S: Chronic radiation enteritis. Clin Oncol 2010;22:70-83.

7 Andreyev J: Gastrointestinal complications of pelvic radiotherapy: are they of any importance? Gut 2005;54:1051-1054.

-8 Andreyev HJ, Vlavianos P, Blake P, Dearnaley D, Norman AR, Tait D: Gastrointestinal symptoms after pelvic radiotherapy: role for the gastroenterologist? Int J Radiat Oncol Biol Phys 2005;62:1464-1471.

-9 Regimbeau JM, Panis Y, Gouzi JL, Fagniez PL: Operative and long term results after surgery for chronic radiation enteritis. Am J Surg 2001;182:237-242.

10 Girvent M, Carlson GL, Anderson I, Shaffer J, Irving M, Scott NA: Intestinal failure after surgery for complicated radiation enteritis. Ann R Coll Surg Engl 2000;82:198-201.

-11 Watt AM, Faragher IG, Griffin TT, Rieger NA, Maddern GJ: Self-expanding metallic stents for relieving malignant colorectal obstruction: a systematic review. Ann Surg 2007;246:24-30.

12 Law WL, Choi HK, Chu KW, Tung HM: Radiation stricture of rectosigmoid treated with self-expanding metallic stent. Surg Endosc 2002;16:1106-1107.

13 Ambrosetti P, Francis K, De Peyer R, Frossard JL: Colorectal anastomotic stenosis after elective laparoscopic sigmoidectomy for diverticular disease: a prospective evaluation of 68 patients. Dis Colon Rectum 2008;51:1345-1349.

14 Hassan C, Zullo A, De Francesco V, Ierardi E, Giustini M, Pitidis A, Taggi F, Winn S, Morini S: Systematic review: Endoscopic dilatation in Crohn's disease. Aliment Pharmacol Ther 2007;26:1457-1464.

15 Hu HT, Shin JH, Kim JH, Park JH, Sung KB, Song HY: Fluoroscopically guided balloon dilation for pharyngoesophageal stricture after radiation therapy in patients with head and neck cancer. AJR Am J Roentgenol 2010;194:1131-1136.

All authors contributed equally to this paper. 